\title{
Application of the selective indocyanine green videoangiography in microsurgical treatment of a craniocervical junction dural arteriovenous fistula
}

\author{
Liyong Sun, MD, Jian Ren, MD, and Hongqi Zhang, MD \\ Department of Neurosurgery, Xuanwu Hospital, Capital Medical University, Beijing, China \\ Craniocervical junction dural arteriovenous fistula (CCJDAVF) is a rare and unique type of intracranial DAVF with com- \\ plex neurovascular anatomy, making it difficult to identify the arterialized vein during operation. The authors report the \\ case of a 50-year-old male who presented with symptoms of venous hypertensive myelopathy. Angiography demon- \\ strated a left CCJDAVF. The fistula was successfully disconnected via a suboccipital midline approach. The selective \\ indocyanine green videoangiography (SICG-VA) technique was applied to distinguish the fistula site and arterialized \\ vein from adjacent normal vessels. Favorable clinical and angiographic outcomes were attained. The detailed operative \\ technique, surgical nuances, and utility of SICG-VA are illustrated in this video atlas.
}

The video can be found here: https://youtu.be/GJYI_jOJQqU.

KEYWORDS craniocervical junction; dural arteriovenous fistula; suboccipital midline approach; indocyanine green videoangiography; video 